\title{
Gradhiva
}

GRADHI

Revue d'anthropologie et d'histoire des arts

Autour de Lucien Sebag

\section{Cannibalisme et mort chez les Guayakis (Achén)}

Pierre Clastres et Lucien Sebag

Salvatore D'Onofrio (éd.)

\section{CpenEdition}

\section{Journals}

Édition électronique

URL : http://journals.openedition.org/gradhiva/511

DOI : 10.4000/gradhiva.511

ISSN : 1760-849X

Éditeur

Musée du quai Branly Jacques Chirac

Édition imprimée

Date de publication : 1 novembre 2005

Pagination : 129-133

ISBN : 2-915-133-10-7

ISSN : 0764-8928

\section{Référence électronique}

Pierre Clastres et Lucien Sebag, « Cannibalisme et mort chez les Guayakis (Achén) », Gradhiva [En ligne], 2 | 2005, mis en ligne le 10 décembre 2008, consulté le 15 septembre 2020. URL : http:// journals.openedition.org/gradhiva/511

Ce document a été généré automatiquement le 15 septembre 2020.

(c) musée du quai Branly 


\title{
Cannibalisme et mort chez les Guayakis (Achén)*
}

\author{
Pierre Clastres et Lucien Sebag
}

Salvatore D'Onofrio (éd.)

\section{NOTE DE L'ÉDITEUR}

Il s'agit d'un article, peu connu et difficilement accessible, signé de Pierre Clastres et Lucien Sebag, «Cannibalisme et mort chez les Guayakis (Achén) » - Achén dans l'original, sûrement une faute de frappe. Y sont relatés les premiers résultats de l'enquête sur un groupe, les Aché-Gaitu, qui étaient entrés en contact avec les Blancs depuis quatre ans. Selon les souvenirs d'Hélène Clastres (communication personnelle), c'est Sebag qui présenta le sujet à la «VI Reunião brasileira de antropologia » qui se tint à São Paulo du 8 au 12 juillet 1963, mais il avait insisté pour que Pierre Clastres le signe avec lui. Le lecteur ne tardera pas à reconnaître l'importance de ces observations ethnographiques et les échos théoriques qu'elles entraînent, d'autant plus que la réflexion sur la nécessité guerrière ou cannibale n'avait été jusqu'alors que rarement menée. Excepté les observations classiques d'André Thevet ou de Jean de Léry au xvie siècle, les articles les plus importants parus sur ces thèmes étaient ceux de Florestan Fernandes, « La guerre et le sacrifice humain chez les Tupinamba » (publié en 1952 dans le Journal de la Société des Américanistes, tome XLI, fasc.1 : 139-221) et de Claude LéviStrauss, « Guerre et commerce chez les Indiens de l'Amérique du Sud » (publié en 1943 dans la revue Renaissance, $\left.\mathrm{n}^{\circ} 1(2): 122-139\right)$. Dans «Cannibalisme et mort chez les Guayakis » les rapports entre cannibalisme et chasse sont posés, avec des distinctions, très originales pour l'époque, entre endocannibalisme et exocannibalisme et ce que l'on pourrait appeler « endocannibalisme ethnique » relevant de l'activité belliqueuse. Le Guayaki tué par un autre Guayaki se trouve par exemple directement identifié à un animal abattu à la chasse, il est traité comme du gibier et son assassin ne peut s'en nourrir. En revanche, c'est la nourriture préférée par les femmes enceintes, car leurs enfants porteront les noms des victimes humaines et des différents gibiers consommés. 
L'acquisition, par les femmes, des noms personnels des hommes et des animaux mangés pendant leur grossesse ouvrait aussi un champ de réflexion très important sur les ressemblances et les différences entre ces types de nourritures, réflexion qui se mêlait également aux conceptions de l'âme multiple, à la valeur accordée aux différentes parties du corps par rapport à l'opposition de genre, au traitement symbolique des os par lequel la distinction entre animalité et humanité est réintroduite. Dans l'article, il est dit que l'enquête doit être complétée par un second séjour de six mois (de juillet en janvier), mais seul Pierre Clastres la poursuivit. Sebag, pour sa part, préféra chercher un autre terrain dans la partie nord-occidentale du Paraguay puis en Bolivie (un choix de ce dernier dont on ne sait rien).

Les photographies que nous avons choisies pour accompagner ce texte représentent un jeune garçon photographié par Sebag qui montre les positions dans lesquelles les AchéKwaré enterraient leurs morts, alors que les Aché-Gaitu, jusqu'à leur rencontre avec les Blancs, les mangeaient - les Paraguayens avaient réuni les deux groupes à Arroyo Morotí sans qu'ils se connaissent auparavant. Sur l'autre photographie, Baipurangi joue de la flûte pour son demi-frère, le petit Brikugi, dont la mort était survenue vers la mijuillet durant la brève absence de Sebag et de Clastres (la photographie a dû être prise dès leur retour de São Paulo). Alors que nul contrôle ne s'exerçait à ce moment sur eux, tous les étrangers (anthropologues, Paraguayens et Aché) étant partis, les Aché-Gaitu mangèrent le petit Brikugi. D'après la remarquable description de Clastres dans sa Chronique, cela se produisit sous la pression de Pikygi, père secondaire de Brikugi, qui pensait ainsi pouvoir guérir de sa maladie.

S. D'O. 
1 Depuis plusieurs siècles, les Guayakis ont été une source d'étonnement pour les voyageurs, les missionnaires et enfin les ethnologues. Dans les cinquante dernières années on a à plusieurs reprises cherché à étudier les différents groupes épars dans la forêt; mais les conditions de stabilité permettant une étude systématique n'avaient encore jamais été réunies; peuple essentiellement nomade, les Guayakis ne se prêtaient pas à l'investigation scientifique en raison de

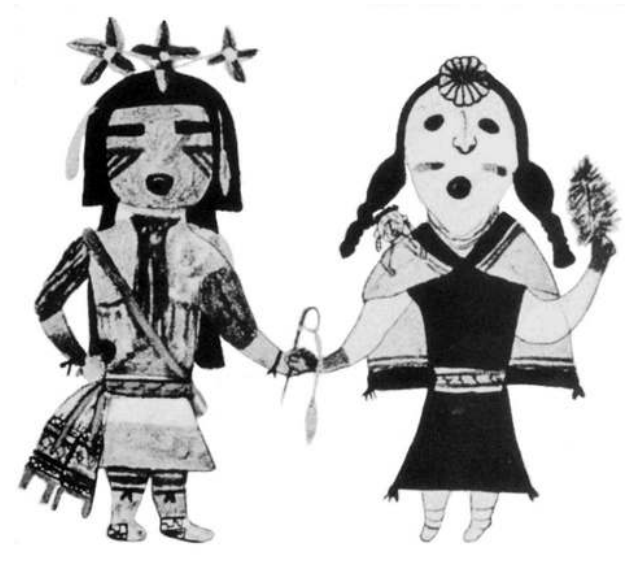
leur extrême mobilité et de leur refus de tout contact; le cas du docteur Vellard qui pendant près de sept mois les poursuivit sans pouvoir les joindre en est une preuve manifeste.

2 La situation a changé depuis quelques années; deux des groupes guayakis, le premier localisé entre San Juan Nepomuceno et Tabai, le second dans la cordillère de l'Yvyturussu, ont été sortis de la forêt par un Paraguayen, Don Manuel Pereira, qui les a pris en charge et les a partiellement fixés. Les conditions étaient alors remplies pour un séjour prolongé, une étude de la langue - indispensable d'ailleurs puisqu'aucun membre de ces deux groupes ne parle le guarani ou l'espagnol - et une analyse aussi précise que possible de leur culture. Ce travail a été amorcé au cours d'un séjour de quatre mois (février-juin 1963) qui doit être complété par un second séjour de six mois (juillet 1963-janvier 1964). Les résultats présentés ici sont donc provisoires ; cependant certains faits, certaines données nous semblent déjà suffisamment établies pour pouvoir être exposées.

3 Les Guayakis sont des nomades, chasseurs et collecteurs, vivant uniquement des ressources de la forêt et ne construisant que des abris rudimentaires qu'ils n'occupent jamais très longtemps. On retrouve chez eux nombre de traits des communautés guarani du Paraguay et du Brésil : il suffit de mentionner la langue (la syntaxe guayaki est très différente de la syntaxe guarani mais la plupart des unités sémantiques peuvent être ramenées soit à l'actuel guarani paraguayen, soit au guarani antique de Montoya), la perforation de la lèvre, la salutation larmoyante, enfin certaines de leurs croyances religieuses (dualité de l'âme, etc.) pour se rendre compte que le rapport est très étroit.

4 Ces différentes caractéristiques sont communes aux deux collectivités étudiées; il n'en est pas de même pour le cannibalisme ; celui-ci semble être le fait d'un seul des deux groupes, celui situé entre San Juan Nepomuceno et Tabai, l'autre pratiquant par contre régulièrement l'inhumation. Ce qui suit concerne donc seulement le groupe de l'Yñaro' ${ }^{1}$.

5 Les membres de ce groupe ont évidemment cessé d'être cannibales lorsqu'ils ont abandonné une partie de leur ancien mode d'existence mais ceci ne remonte qu'à cinq ans; antérieurement tous les morts étaient mangés, le dernier cas mentionné ayant approximativement eu lieu il y a neuf ans (la datation en est très aisée, on verra pourquoi par la suite), aucun Indien n'étant mort entre ce moment et celui de la sortie de la tribu. Il résulte de ceci que lorsqu'on parle de cannibalisme il ne s'agit nullement de «on-dit» mais d'une réalité que tous les individus ont vécu; de plus, psychologiquement, les Guayakis sont encore cannibales; à plusieurs reprises ils nous ont affirmé leur désir de manger de la chair humaine et une de nos jeunes 
informatrices a récemment rêvé que son "Jware" (homme qui a assisté à l'accouchement et massé la tête du nouveau-né) étant mort, elle le faisait cuire et s'en nourrissait. Cette absence de réticences nous a permis de mener notre enquête en interrogeant la totalité des membres adultes du groupe; chaque cas de cannibalisme nous a été raconté plusieurs fois; nous les avons souvent recueillis indépendamment l'un de l'autre et toutes les précautions ont été prises pour exclure le malentendu, l'invention, les conséquences de la fantaisie personnelle d'un informateur.

Jeune Aché arborant ses peintures corporelles, Arroyo Morotí, 1963

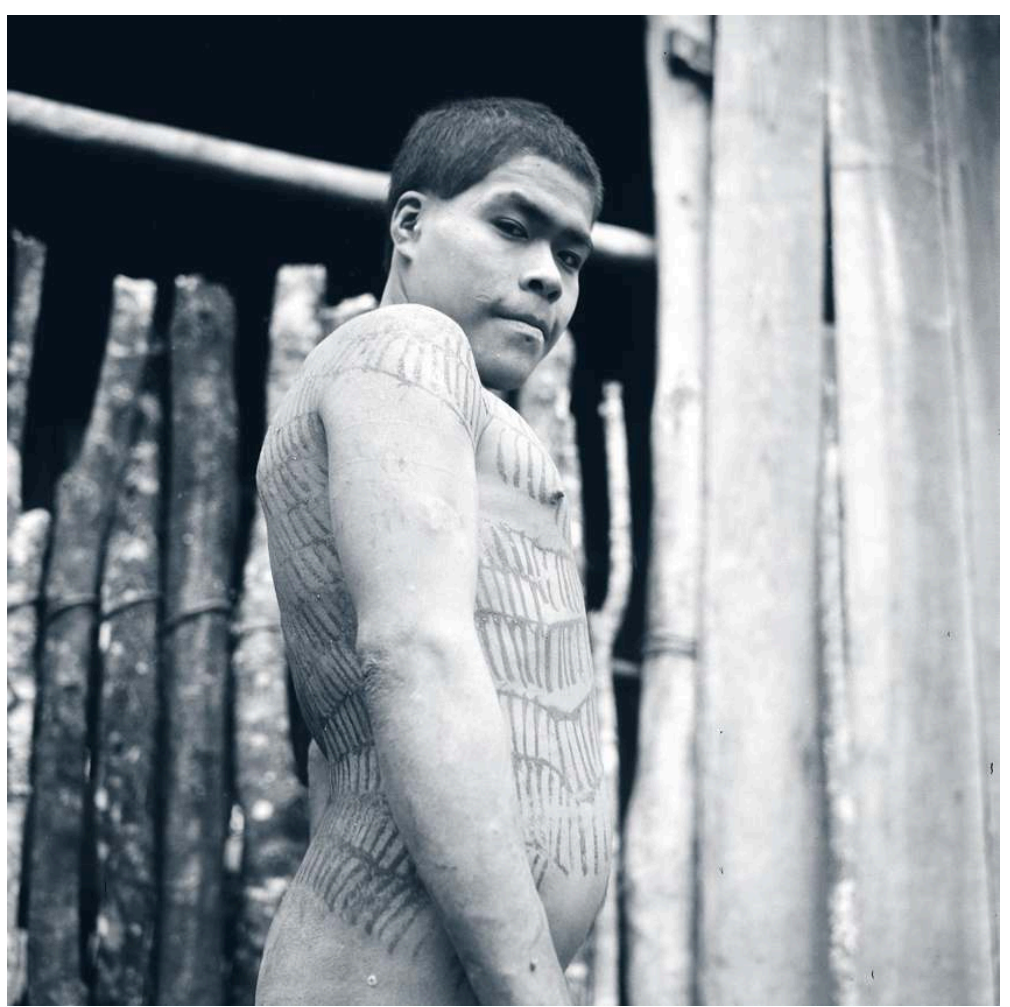

(C) Laboratoire d'anthropologie sociale, fonds Sebag

6 Les morts sont donc mangés quelle que soit la manière dont ils ont perdu la vie ; mais un recensement des principaux types de mort fraie la voie à une compréhension de la culture guayaki : certains des membres disparus du groupe ont succombé à la maladie ; d'autres - le nombre en est plus élevé - ont été tués ; ils l'ont été soit par un animal soit par des Guayakis du même groupe; agissant ainsi, ces derniers obéissaient ou à des préoccupations socio-économiques (mise à mort des vieillards incapables de pouvoir marcher dans la forêt et d'assurer leur subsistance) ou à des obligations d'ordre religieux (nécessité de venger un autre mort pour en quelque sorte apaiser son âme). Ce qui nous donne le diagramme suivant: 


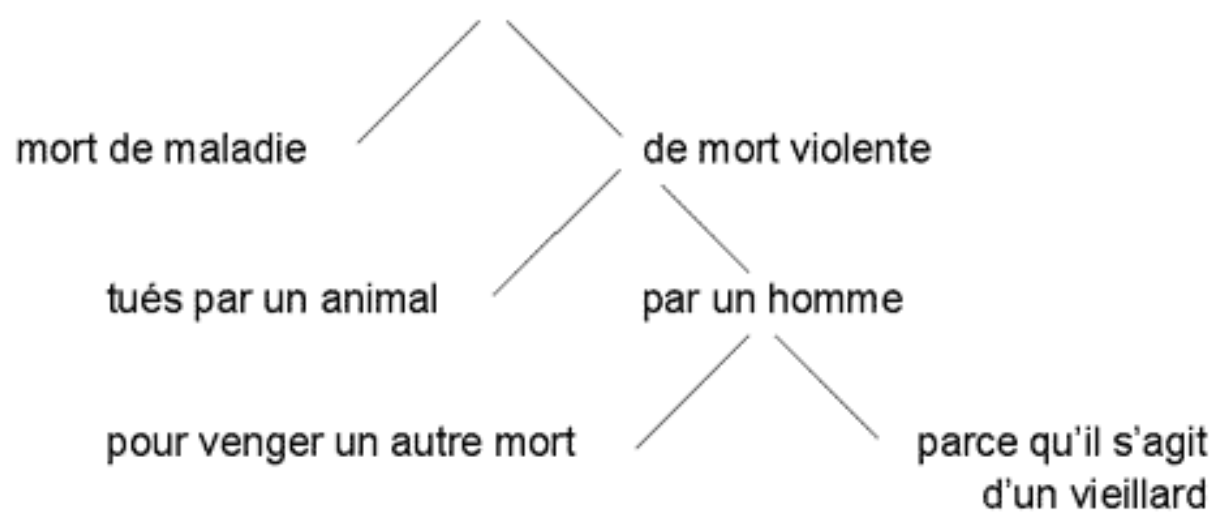

7 Ces types de décès sont très différents les uns des autres, mais il serait aisé de montrer que le système de croyances guayaki permet d'établir certaines équivalences (sauf en ce qui concerne le meurtre des vieillards qui appartient à un autre registre) entre ces diverses classes. La maladie résulte toujours de la consommation de la chair de certains animaux : mourir du « baiwa » est le risque que l'on court en se nourissant de tatous, de coatis ou de miel d'abeille ; l'animal tué se venge ; qu'il s'agisse d'une vengeance ressort $\mathrm{du}$ fait que d'autres animaux ne provoquent pas de maladie mais impliquent des dangers d'un autre ordre : après avoir mangé du carpincho on ne risque pas de voir sa santé s'altérer mais on pourra être dévoré par le jaguar, animal dont l'une des fonctions est de venger le carpincho. Ainsi les Guayakis rapprochent-ils directement les maladies mortelles et certains types de mort violente. Or ces animaux vengeurs sont aussi ceux qui portent l'âme du Guayaki disparu ; la surgie du jaguar sera donc liée à une double finalité ; elle rétablira l'équilibre dans le domaine animal comme dans le domaine humain; pour expliquer son intervention ou pourra recourir à plusieurs interprétations : ainsi la mort d'un jeune homme, Brevi Purangi, ainsi dévoré, était rapportée au fait qu'il avait ri en mangeant du fourmilier, violation de tabou que le jaguar punit; mais par ailleurs ce dernier était identifié à l'ancienne épouse de Brevi Purangi, décédée depuis peu et venant reprendre son époux après s'être en quelque sorte incarnée dans un jaguar. Il s'agissait là d'une vengeance directe accomplie par l'intéressé lui-même. Mais lorsqu'il tue un enfant après la mort d'un adulte, dans le but de venger celui-ci, le Guayaki ne fait rien d'autre que rétablir un équilibre que la mort vient de compromettre. Sans entrer dans les détails il faut noter qu'on a parfois de véritables séries qui ne sont closes qu'après le meurtre de trois ou quatre personnes. Ainsi le père de Brevi Purangi a vengé celui-ci en tuant de ses femmes; cet assassinat a entraîné celui d'une petite fille qui elle-même a été vengée par la mort d'une autre petite fille. Une telle chaîne ne se trouve formée qu'après qu'une dernière tentative de meurtre n'a pas été menée jusqu'à son terme, les femmes du groupe apaisant par leurs massages et leurs implorations le meurtrier en puissance ${ }^{2}$. Les victimes sont généralement de sexe féminin et il s'agit dans la plupart des cas de petites filles orphelines; mais parfois ce sont des garçons ou même des hommes qui ont été tués. Cette prédominance d'un sexe sur l'autre découle de la préférence marquée que les Guayakis ont pour les enfants de sexe masculin; la naissance d'une fille n'est jamais accueillie avec joie et certaines conduites ont pour but explicite de la rendre impossible.

8 La cause de la mort n'empêche cependant jamais qu'on se nourrisse du corps; lorsque le décès a eu lieu tard dans l'après-midi ou au cours de la nuit le cadavre est recouvert de fougères ; il est en effet interdit de manger de la chair humaine à la nuit tombante 
sous peine d'être malade (la règle est la même pour de nombreux animaux); le lendemain le corps est découvert et découpé ; un gril (buta) est dressé et les différentes parties du corps, bras, jambes, tronc, sont posées et roties sur ce gril ; ce dernier, qu'on utilise aussi pour certains animaux, est identique à celui dont les Tupinamba se servaient pour la chair humaine; d'après les descriptions que nous avons obtenues, le gril sur lequel reposaient les différentes parties du corps humain présentait exactement le même aspect que celui des Tupinamba tel que nous le connaissons d'après les dessins de l'époque.

Rôtie, la viande est mangée par l'ensemble des membres du groupe, enfants et vieillards compris; seuls sont exclus du festin le père et la mère de la victime et son conjoint véritable (imé été) ; le second type d'époux (japétyva) n'est par contre soumis à aucun interdit. Pour les fils et filles la situation est moins claire; certains d'entre eux ont mangé leurs parents; d'autres par contre s'y sont refusé; il semble qu'ici interviennent des préférences individuelles. Dans le cas où le mort a été tué par un autre Guayaki, ce dernier ne peut pas manger sa victime et son père et sa mère se trouvent dans la même situation. Il ne s'agit pas là d'un statut particulier : le meurtrier d'Aché (Brupiatygi) agit exactement comme un quelconque chasseur ; celui-ci ne peut jamais se nourrir des animaux qu'il a tués et la même interdiction vaut pour ceux qui lui ont donné la vie ; la viande qu'il apporte est destinée à sa femme, à ses enfants et aux autres membres de la tribu; lui-même attend pour consommer de la viande d'en recevoir de ses compagnons. De ce point de vue le Guayaki assassiné se trouve directement identifié à un animal tué à la chasse.

De même la consommation de la chair humaine ne semble pas présenter d'importantes différences par rapport à celle de la chair animale; les tabous qui y sont attenants (ne pas rire, ne pas manger couché ou lorsque la nuit est tombée) sont du même ordre dans les deux cas. Ce qui suit le repas est par contre plus spécifique: une fois la viande consommée, les os du cadavre sont brisés, sucés, puis jetés dans le feu; le crâne est pilé et les morceaux en sont brûlés. C'est là un acte d'une extrême importance car la fumée en s'élevant permettra la montée de l'âme dans sa demeure céleste. Le problème se complique ici en raison de la croyance à l'existence d'âmes multiples; celles-ci peuvent se laisser regrouper sous deux grands principes ${ }^{3}$ : d'une part l'âme céleste est bénéfique (Owé), de l'autre l'âme tellurique (Ianwé) vivant dans la forêt et associée à tous les esprits mauvais ; cette dernière représente un danger permanent pour le Guayaki : elle peut venir le frapper pendant son sommeil, elle peut pénétrer dans son ventre et provoquer sa mort (certaines maladies sont rapportées à Ianwé), elle peut chercher à s'emparer de ceux qui lui étaient proches durant sa vie terrestre. On ne prendra donc jamais assez de précautions : ainsi quittera-

$\mathrm{t}$-on le campement après un décès pour s'éloigner des lieux où Ianwé peut séjourner. La quémation des os semble répondre à une double exigence : permettre la montée d'Owé au ciel, écarter Ianwé. Il n'y a évidemment rien de pareil en ce qui concerne les animaux; ceux-ci n'ont pas d'âme et les dangers courus après leur consommation ne sont pas du même ordre; les précautions prises à leur égard sont donc tout à fait différentes. Sous cet angle animalité et humanité se distinguent profondément.

11 La quémation des os a donc une signification religieuse, explicitement attestée chaque fois qu'un cas d'anthropophagie est relaté. Il n'en est pas de même du cannibalisme luimême : jusqu'à présent seule a été mise en avant la valeur alimentaire d'un tel acte. En ce qui concerne l'endocannibalisme il nous a été dit que les morts étaient mangés parce 
que leur chair était bonne; en ce qui concerne l'exocannibalisme, chaque fois que nous avons demandé pourquoi ils avaient tué des Guayakis d'un autre groupe, il nous a été répondu: «pour les manger ». La question reste donc ouverte et seule une meilleure connaissance de la langue permettra sur ce point d'arriver à une certitude.

Ressemblances et différences entre le statut accordé à la nourriture animale et celui de la nourriture humaine apparaissent clairement dans une autre perspective. Les noms personnels des Guayakis sont empruntés au règne animal; l'enfant porte le nom de tous les animaux que sa mère a mangés alors qu'elle était enceinte de lui ; il en résulte que chaque individu peut être désigné par un très grand nombre de termes, dans certains cas près d'une vingtaine ${ }^{4}$; ce qui exclut toute ressemblance entre l'animal patronymique et l'individu en question. Alors que chez les Apapokuva il s'agit d'une véritable identification entre tel homme et tel animal dont il porte le nom ${ }^{5}$ (ce qui implique que cet animal soit unique), rien de tel n'apparait chez les Guayakis : le même enfant sera chevreuil, jaguar, oiseau, poisson, singe, etc. et à aucun moment nous n'avons vu lui attribuer les qualités et les défauts de ces êtres. Or la consommation de la chair humaine intervient identiquement dans l'obtention du nom personnel: je porterai le nom des hommes que ma mère a mangés lorsqu'elle était enceinte de moi. Mais il ne s'agit plus alors d'une simple attribution extérieure mais d'une véritable réincarnation. L'enfant possède l'âme (Owé) de celui que sa mère a mangé : ainsi Kybwyragi, l'un de nos meilleurs informateurs, possède l'âme de Jurupurangi, un Aché d'un autre groupe tué au cours d'une expédition guerrière, puis mangé ; ceci parce que sa mère a participé au festin. De cela il résulte que la majorité des membres du groupe de l'Yñaro ont non seulement des "bykwa » (terme qui désigne le nom ainsi obtenu) animaux mais aussi des « bykwa » humains. En ce dernier cas c'est l'âme du mort que je possède. On comprend dès lors pourquoi la datation est aisée : un enfant de huit ans ayant un «bykwa » humain me permet de savoir qu'un homme a été mangé, au plus tard neuf mois avant sa naissance.

13 Lorsqu'on parle d'âme, celle-ci ne doit pas être comprise comme un principe unique et substantiel se transmettant d'individu à individu ; le même mort peut se réincarner en plusieurs personnes; il suffit que leurs mères aient été enceintes simultanément. Inversement un enfant peut avoir les âmes de tous les membres du groupe qui sont morts pendant sa gestation; comme pour les animaux on se situe là dans un domaine indéfini que rien ne vient fermer sinon une limitation de fait.

14 Les femmes enceintes occupaient donc toujours une place de choix au cours des repas anthropophagiques; et [si] elles se rappellent avec précision les morceaux qu'elles ont mangés, c'est qu'enfants, adultes, vieillards, hommes, femmes ne reçoivent pas les mêmes parties du corps d'un animal ou d'un homme, l'âge ou le sexe intervenant dans la répartition. Cependant le cas de la chair humaine est particulier: les femmes enceintes ont généralement mangé, lorsqu'il s'agissait d'un homme, le pénis de celui qui venait de mourir ; ce morceau leur était expressément réservé et il pouvait même être partagé lorsque plusieurs femmes attendaient simultanément un enfant. Cette consommation de la verge, qui établit une équivalence entre sexualité et alimentation, est rapportée au désir d'avoir des garçons. Par contre lorsque c'est une femme qui était mangée, le bas du tronc et la partie correspondant au vagin étaient enterrés dans le but explicite de prévenir la naissance d'une fille.

De fait mort et cannibalisme nous conduisent au cœur même de la culture du groupe de l'Yñaro ; toutes les formes de conduites et de croyances se rattachent d'une manière ou 
d'une autre à la consommation de la chair humaine: place de l'activité chasseresse, relation au monde animal, signification de la transmission du nom, difficultés soulevées par la dualité de l'âme. Ces problèmes demandent à être éclaircis pour que le cannibalisme apparaisse dans son sens plein. Les données que nous venons de présenter sont fragmentaires et attendent d'être enrichies et précisées par notre travail ultérieur; de plus l'étude des deux autres groupes encore sauvages permettra, s'ils sont cannibales, de posséder plusieurs variantes des mêmes faits et de mieux apprécier le sens du matériel obtenu à propos du groupe de l'Yñaro.

\section{NOTES}

*. Revista do Museu Paulista n.s., XIV 1964 : 174-171. L'astérisque du titre renvoie à la note « autodénomination des deux groupes guayakis étudiés ».

1. Dénomination purement géographique donnée par León Cadogan, « Algunos textos guayaki de l'Yñaro », Asunción, 1961.

2. Une dizaine de ces vengeances en chaîne nous ont été relatées ; elles varient certes dans la manière mais obéissent toutes au même principe.

3. En ce domaine la terminologie guayaki reste confuse ; nous ne pouvons être là que très schématiques.

4. Les Siriono ont un système analogue et portent des noms d'animaux ; mais ce sont ceux que le père a tués immédiatement après la naissance de l'enfant. Cf. Holmberg, « Nomads of the long bow $»$.

5. Cf. Curt Nimuendaju, «Leyenda de la creación y juicio final del mundo como fundamento de la religión de los Apapokuva-Guarani », San Pablo, 1944. Le nom animal, qui n'est d'ailleurs pas le seul, est obtenu d'une tout autre manière. 